an initial screen with a panel of 791 antibodies. Proteomics 2003; 3(6): 991-1002.

Disclosure of Interests: None declared

DOI: 10.1136/annrheumdis-2019-eular.3539

\section{SAT0071 ANALYSIS OF CHRONOLOGICAL CHANGES IN JAPANESE VERSION OF HEALTH ASSESSMENT QUESTIONNAIRE SCORE AND FACTORS ASSOCIATED WITH J-HAQ REMISSION AT 5 YEARS AFTER DISEASE ONSET IN PATIENTS WITH RHEUMATOID ARTHRITIS USING THE IORRA COHORT}

Mai Abe ${ }^{1}$, Eiichi Tanaka ${ }^{1}$, Eisuke Inoue ${ }^{1,2}$, Mika Kawano $^{1}$, Eri Sugano ${ }^{1}$, Naohiro Sugitani ${ }^{1,3}$, Kumiko Saka ${ }^{1}$, Yoko Shimizu ${ }^{1}$, Moeko Ochiai ${ }^{1}$, Rei Yamaguchi ${ }^{1}$, Naoki Sugimoto ${ }^{1}$, Katsunori Kkari $^{1}$, Ayako Nakajima ${ }^{1,3}$ Atsuo Taniguchi ${ }^{1}$, Hisashi Yamanaka ${ }^{1}{ }^{1}$ Tokyo Women's Medical University, Department of Rheumatology, Tokyo, Japan; ${ }^{2}$ St. Marianna University School of Medicine, Division of Medical Informatics, Kanagawa, Japan; ${ }^{3}$ Mie Univercity Hospital, Center for Rheumatic Diseases, Mie, Japan

Background: Recent advances in rheumatoid arthritis (RA) treatment including the introduction of biologics have greatly affected treatment strategies for RA, achieving remission as a realistic treatment target. However, few reports have been concerned chronological changes in longterm physical dysfunction among large numbers of RA patients in daily practice.

Objectives: To evaluate chronological changes in Japanese version of Health Assessment Questionnaire score (J-HAQ) score and J-HAQ remission rates at 5 years after RA onset using the Institute of Rheumatology, Rheumatoid Arthritis (IORRA) cohort.

Methods: RA patients who developed RA between 2000 and 2010 and who first visited our hospital during the year of RA onset were divided into two groups: 1) former onset group (RA onset between 2000 and 2005) and 2) recent onset group (RA onset between 2006 and 2010). JHAQ scores and J-HAQ remission rates at baseline and at 5 years after onset were investigated for each group, and factors associated with $\mathrm{J}$ $\mathrm{HAQ}$ remission after 5 years were assessed by logistic regression analysis. Methotrexate (MTX), corticosteroid (steroid) and biologic DMARDs user (bDMARDs) was defined as the patients if they were used each medication during the observation period.

Results: The former onset group and recent onset group included 357 and 291 RA patients, respectively. For the former onset group, the average J-HAQ score/J-HAQ remission rate at baseline and 5 years after the onset were $0.659 / 54.6 \%$ and $0.430 / 71.4 \%$, respectively. The recent onset group showed significant improvements relative to the former onset group in J-HAQ score/J-HAQ remission rates at baseline and 5 years of $0.705 /$ $52.2 \%$ and $0.316 / 78.4 \%$, respectively $(\mathrm{p}=0.011 / 0.044)$. The percentage of MTX and bDMARDs users was significantly higher in the recent onset group (former vs. recent onset group; MTX: $70.9 \%$ vs. $86.6 \%$ [p $<0.0001]$; bDMARDs: $5.3 \%$ vs. $23.0 \%$ [p <0.0001]). Significant factors associated with achieving J-HAQ remission at 5 years after RA onset were: patients in the recent onset group $(p<0.001)$, male $(p<0.001)$, younger $(p<0.001)$, lower J-HAQ score $(p<0.001)$ at baseline, and non-steroid user $(p<0.001)$. Conclusion: In daily practice, J-HAQ scores for RA patients remarkably improved with recent advances in RA treatment strategies. To achieve $\mathrm{J}$ $\mathrm{HAQ}$ remission at 5 years of RA onset, beginning treatment in the early disease stage is needed to prevent deterioration of J-HAQ and treatments that avoid steroid use appear to be important.

Acknowledgement: We thank all patients who participated in the IORRA survey and all of the members of the Institute of Rheumatology, Tokyo Women's Medical University, for the successful management of the IORRA cohort.

Disclosure of Interests: Mai Abe: None declared, Eiichi Tanaka Speakers bureau: Abbvie, Asahi Kasei pharma co., Bristol Myers Squibb, Chugai Pharmaceutical, Daiichi Sankyo Co., Eisai Pharmaceutical, Janssen Pharmaceutical K.K., Nippon Kayaku, Pfizer, Takeda Pharmaceutical, Taisho Toyama Pharmaceutical Co., and UCB Pharma., Eisuke Inoue: None declared, Mika Kawano: None declared, Eri Sugano: None declared, Naohiro Sugitani: None declared, Kumiko Saka: None declared, Yoko Shimizu: None declared, Moeko Ochiai: None declared, Rei Yamaguchi: None declared, Naoki Sugimoto: None declared, Katsunori Ikari: None declared, Ayako Nakajima Grant/research support from: Asahi Kasei pharma co., Chugai Pharmaceutical, Daiichi Sankyo Co., Pfizer, Kissei Pharmaceutical Co., and Mitsubishi Tanabe Pharma Corporation., Atsuo Taniguchi: None declared, Hisashi Yamanaka Grant/research support from: AbbVie, Eisai, Bristol-Meyers, Novartis, Behringer, Astellas, Kaken,
Nippon-Shinyaku, Pfizer, UCB, Ayumi, Ono, Daiichi-Sankyo, TaisyoToyama, Takeda, Tanabe-Mitsubishi, Chugai, Teijin Pharma, Torii, YLbio, Speakers bureau: Bristol-Meyers, Astellas, Pfizer, Daiichi-Sankyo, Takeda Tanabe-Mitsubishi, Chugai, Teijin Pharma, YLbio DOI: 10.1136/annrheumdis-2019-eular.3597

\section{SAT0072 EVALUATION OF RHEUMATOID ARTHRITIS TREATMENTS AND JOINT OUTCOMES IN RHEUMATOID ARTHRITIS-ASSOCIATED INTERSTITIAL LUNG DISEASE}

Evo Alemao ${ }^{1}$, Tracy Doyle ${ }^{2}$, Jeffrey Sparks ${ }^{2}$, Aarti Rao ${ }^{3}$, Yogesh Saini ${ }^{3}$, Christine lannaccone ${ }^{2}$, Michael E. Weinblatt ${ }^{2}$, Nancy Shadick ${ }^{2} .{ }^{1}$ Bristol-Myers Squibb, Princeton, United States of America; ${ }^{2}$ Brigham and Women's Hospital, Boston, United States of America; ${ }^{3} \mathrm{Mu}$ Sigma, Bangalore, India

Background: RA-associated interstitial lung disease (RA-ILD) is an extraarticular manifestation of RA and is one of the leading causes of death in patients (pts) with RA. ${ }^{1}$ Previous studies have indicated that clinical factors such as age, sex, smoking and autoantibody positivity are strongly associated with RA-ILD. ${ }^{2}$ There is also evidence of active RA being related to increased risk for clinically apparent ILD. ${ }^{3}$ However, there are limited data on how pts with RA-ILD are managed for their joint conditions and joint outcomes.

Objectives: To evaluate RA treatment patterns in pts with subclinical and clinical RA-ILD compared with pts with RA without ILD, and to assess joint disease activity at baseline and change in disease activity by treatment in all cohorts.

Methods: Data from adult pts with RA enrolled in a longitudinal sequential RA registry were analysed. Pts in the registry were evaluated annually by a rheumatologist for disease activity and treatment, and semiannually on multiple clinical patient-reported outcomes (PROs) and resource utilisation parameters. Pts with chest computed tomography (CT) scans performed to evaluate clinical indications for ILD and with blood samples were included in this analysis. Pts with chest CT scans that were indeterminate for ILD were excluded from the study. Pts were then divided into two mutually exclusive groups: non-ILD RA pts and RA-ILD pts. RA-ILD pts were further divided into subclinical and clinically evident ILD. Date of chest CT scan was considered the index date. The two cohorts were compared using descriptive statistics to summarise baseline differences in demographics, disease activity measures, serostatus and treatments. Kruskal-Wallis test for continuous variables and chi-square test for categorical variables were performed, with two-sided significance level of 0.05 . Multivariable linear regression was used to evaluate change from baseline to 12 months in joint disease activity for pts with available data at baseline and follow-up.

Table 1. Baseline characteristics and treatments by RA-ILD groups

\begin{tabular}{|c|c|c|c|c|}
\hline & No ILD (n=29) & ILD (n=46) & $\begin{array}{c}\text { Subclinical ILD } \\
(n=29)\end{array}$ & $\begin{array}{c}\text { Clinically } \\
\text { evident ILD } \\
\text { (n=17) }\end{array}$ \\
\hline Age, years, mean (SD) & $53.0(12.2)$ & $66.9(9.5)^{*}$ & $68.3(9.6)^{x}$ & $64.6(9.1)^{x}$ \\
\hline Duration of RA, years, mean (SD) & $16.9(11.6)$ & $23.0(13.3)^{*}$ & $24.4(14.4)^{*}$ & $20.6(11.0)$ \\
\hline Female, $\mathbf{n}(\%)$ & $28(96.6)$ & $36(78.3)^{*}$ & $23(79.3)$ & $13(76.5)$ \\
\hline History of smoking, $n(\%)$ & $12(41.4)$ & $29(63.0)$ & $20(69.0)$ & $9(52.9)$ \\
\hline RF positive, $n(\%)$ & $\begin{array}{c}\mathrm{n}=27 \\
18(66.7)\end{array}$ & $39(84.8)$ & $24(82.8)$ & $15(88.2)$ \\
\hline RF titre, IU/mL, mean (SD) & $\begin{array}{c}\mathrm{n}=27 \\
105.0(177.9)\end{array}$ & $338.7(618.0)^{*}$ & $423.2(759.3)^{*}$ & $194.7(174.1)^{*}$ \\
\hline ACPA positive, $n(\%)$ & $15(51.7)$ & $40(87.0)^{*}$ & $26(89.7)^{*}$ & $14(82.4)^{*}$ \\
\hline ACPA titre, IU/mL, mean (SD) & $87.1(130.0)$ & $212.8(191.9)^{x}$ & $183.7(149.3)^{*}$ & $262.6(245.7)^{*}$ \\
\hline CDAl score, mean (SD) & $\begin{array}{c}n=21 \\
21.1(15.6)\end{array}$ & $\begin{array}{c}n=39 \\
26.3(14.5)\end{array}$ & $\begin{array}{c}n=25 \\
26.3(14.6)\end{array}$ & $\begin{array}{c}n=14 \\
26.4(14.9)\end{array}$ \\
\hline mHAQ score, mean (SD) & $\begin{array}{c}n=27 \\
0.4(0.4)\end{array}$ & $\begin{array}{c}n=43 \\
0.6(0.5)\end{array}$ & $\begin{array}{c}n=27 \\
0.5(0.5)\end{array}$ & $\begin{array}{c}n=16 \\
0.6(0.5)\end{array}$ \\
\hline Previous TNFi bDMARD, $n(\%)$ & $2(6.9)$ & $7(15.2)$ & $2(6.9)$ & $5(29.4)$ \\
\hline Current TNFi bDMARD, $n(\%)$ & $12(41.4)$ & $16(34.8)$ & $10(34.5)$ & $6(35.3)$ \\
\hline Previous non-TNFi, $n(\%)$ & $0(0.0)$ & $3(6.5)$ & $0(0.0)$ & $3(17.6)^{x}$ \\
\hline Current non-TNFi, $n(\%)$ & $3(10.3)$ & $5(10.9)$ & $2(6.9)$ & $3(17.6)$ \\
\hline Previous steroids, $\mathrm{n}(\%)$ & $16(55.2)$ & $31(67.4)$ & $19(65.5)$ & $12(70.6)$ \\
\hline Current steroids, $\mathbf{n}(\%)$ & $6(20.7)$ & $22(47.8)^{*}$ & $13(44.8)^{x}$ & $9(52.9)^{x}$ \\
\hline Previous MTX, $n(\%)$ & $3(10.3)$ & $5(10.9)$ & $4(13.8)$ & $1(5.9)$ \\
\hline Current MTX, n (\%) & $7(24.1)$ & $17(37.0)$ & $14(48.3)$ & $3(17,6)$ \\
\hline \multicolumn{5}{|l|}{$p<0.05$ vs no ILD } \\
\hline $\begin{array}{l}\mathrm{ACPA}=\text { anti-citrullinated pr } \\
\mathrm{mHAQ}=\text { modified } \mathrm{HAQ} ; \mathrm{TN}\end{array}$ & bDM & & & \\
\hline
\end{tabular}


Results: 75 pts with chest CT scans were included in the analysis. Of these, $38.7 \% \quad(n=29)$ were non-ILD RA and $61.3 \% \quad(n=46)$ had some manifestation of RA-ILD. Of the RA-ILD cohort, $63.0 \%(n=29)$ and $37.0 \%$ $(\mathrm{n}=17)$ were classified as subclinical and clinically evident RA-ILD, respectively. At the time of chest CT scan, RA-ILD (vs non-ILD RA) pts were older with longer disease duration, a greater proportion were male and had higher anti-citrullinated protein antibody and RF titres (Table 1). In terms of RA treatments, a significantly greater proportion of RA-ILD (vs non-ILD RA) pts were on corticosteroids (CS; $47.8 \%$ vs $20.7 \%$ ) and a significantly greater proportion of clinically evident RA-ILD (vs non-ILD RA) were on non-TNF inhibitor (TNFi) biologics. RA-ILD (vs non-ILD RA) pts had numerically higher joint disease activity and modified HAQ score at baseline (Table 1). However, the change in joint disease activity and PROs in RA-ILD pts was numerically greater vs non-ILD RA pts. In a multivariable analysis, RA-ILD status did not impact change in joint disease activity, but baseline joint disease activity was significantly associated with reduction in joint disease activity at 12 months (Table 2).

Conclusion: RA-ILD pts compared with non-ILD RA pts in clinical practice are more likely to be managed with CS therapy and non-TNFi bDMARDs. The improvement in joint disease activity was similar between RA-ILD and non-ILD RA pts. The analysis was limited by small sample size; further studies with larger pt numbers are required to confirm the findings.

Table 2. Multivariable analysis of change in disease activity (CDAl)

\begin{tabular}{|l|c|c|}
\hline Parameter & Estimate & $\mathbf{9 5 \%} \mathbf{~ C l}$ \\
\hline Intercept & 0.8 & $(-12.5,14.0)$ \\
\hline No ILD (vs ILD) & -3.9 & $(-12.8,5.1)$ \\
\hline RA duration, years & -0.1 & $(-0.5,0.3)$ \\
\hline Baseline CDAl score* & 0.4 & $(0.0,0.7)$ \\
\hline No CS (vs CS) & -3.6 & $(-13.5,6.2)$ \\
\hline $\begin{array}{l}{ }^{*} \mathrm{p}<0.05 \text { vs no ILD } \\
\mathrm{CS}=\text { corticosteroids; ILD=interstitial lung disease }\end{array}$ \\
\hline
\end{tabular}

\section{REFERENCES}

[1] Doyle JT, et al. Am J Respir Crit Care Med 2015;191:1403-12.

[2] Sparks JA, et al. Arthritis Care Res (Hoboken) 2016;68:753-62.

[3] Sparks JA, et al. Arthritis Rheumatol 2018; 70(suppl 10):abstract 884.

Disclosure of Interests: Evo Alemao Shareholder of: Bristol-Myers Squibb, Employee of: Bristol-Myers Squibb, Tracy Doyle Grant/research support from: Receive research support from Bristol-Myers Squibb and I'm involved in a clinical trial funded by Genentech., Jeffrey Sparks Grant/ research support from: Bristol-Myers Squibb, Amgen, Consultant for: Optum, Aarti Rao Consultant for: Bristol-Myers Squibb, Yogesh Saini Consultant for: Bristol-Myers Squibb, Christine lannaccone: None declared, Michael E. Weinblatt Shareholder of: Stock option: CanFite, Lycera, Scipher, Inmedix, Grant/research support from: Crescendo Bioscience, Bristol Myers Squibb, Sanofi, Consultant for: AbbVie, Amgen, Bristol-Myers Squibb, CanFite, Corrona, Crescendo, GlaxoSmithKline, Gilead, Horizon, Lilly, Lycera, Merck, Novartis, Pfizer, Roche, Samsung, Scipher, Set Point, Nancy Shadick Grant/research support from: Bristol-Myers Squibb, Sanofi/ Regeneron, Crescendo Biosciences, Mallinckrodt, Amgen, Consultant for: Consulting work for Bristol-Myers Squibb for under $\$ 10,000$.

DOI: 10.1136/annrheumdis-2019-eular.1766

\section{SAT0073 \\ STAT3/STAT5 BALANCE AS A BIOMARKER IN RA: CTLA4-IG AND T CELL DIFFERENTIATION THROUGH STAT SIGNALING}

Stefano Alivernini ${ }^{1}$, Barbara Tolusso ${ }^{2}$, Anna Laura Fedele ${ }^{2}$, Clara DI Mario ${ }^{3}$, Luca Petricca $^{2}$, Maria Rita Gigante ${ }^{1}$, Gianfranco Ferraccioli ${ }^{3}$, Elisa Gremese ${ }^{1}$. ${ }^{1}$ Fondazione Policlinico Universitario A. Gemelli IRCCS - Università Cattolica del Sacro Cuore, Division of Rheumatology, Rome, Italy, ${ }^{2}$ Fondazione Policlinico Universitario A. Gemelli IRCCS, Division of Rheumatology, Rome, Italy; ${ }^{3}$ Università Cattolica del Sacro Cuore, Division of Rheumatology, Rome, Italy

Background: Regulatory $\mathrm{T}$ cells (Treg) play suppressive functions and are modulated by Abatacept (CTLA4-lg). Limited data are available on CTLA4-Ig effect on Treg population in Rheumatoid Arthritis (RA)

Objectives: The aim of the study was to analyze if STAT3/STAT5 expression in CD4+ $T$ cells in peripheral blood (PB) of RA patients at baseline predicts response to Abatacept treatment (after 12 months follow-up).
Methods: Early RA (ERA) and long-standing RA (LS-RA) patients with conventional DMARDs insufficient response were enrolled in this observational, investigative, monocentric, non-randomized, no profit study, and treated with CTLA4-lg in combination with methotrexate. Each enrolled RA patients underwent peripheral blood sampling and CD4+ cells isolation using magnetic micro-beads at baseline and after 6-12 months follow-up. Flow cytometric analysis (FACS) for CD4 positive cells phenotype was performed to assess T-regulatory cells (Treg) as CD4+/CD25+/CD127and CD4+/CD25+/Foxp3+, respectively. STAT3/STAT5 gene expression on CD4+ cells was performed by RT-PCR for each enrolled patient at every time-point follow-up. Low disease activity (LDA) and disease remission (DAS) achievement were assessed at 6 and 12 months follow-up (FU), respectively.

Results: A total of 35 patients were enrolled in the study (16 ERA and 19 LS-RA, respectively). At baseline, ERA and LS-RA did not differ based on clinical parameters. Eight (22.9\%) withdrew from the study because of treatment failure $(n=6)$, severe infection $(n=1)$ and death $(n=1)$. LDA or DAS remission within twelve months follow-up were achieved in $28 / 34(82.4 \%)$ and $16 / 34$ (47.1\%) patients, respectively, without any significant difference among ERA and LS-RA. There were no significant differences in the demographic and clinical characteristics of RA patients at study based on LDA or DAS remission status achievement within 12 months FU, even stratifying patients based on disease duration. FACS analysis showed CD4+/CD25+/CD127- and CD4+/CD25+/ Foxp3+ cells decrease during CTLA4-Ig treatment $(p=0.01$ and $p=0.02$ respectively after 12 months $F U$ ), despite disease duration. RT-PCR revealed that $\mathrm{PB}$ CD4+ cells of RA patients achieving LDA, but not DAS remission, after CTLA4-Ig treatment have significantly lower endogenous expression of STAT3 and STAT5 compared to RA patients not achieving this outcome $(p=0.03$ and $p<0.001$, respectively). Moreover, baseline STAT3/STAT5 ratio in PB CD4+ cells of RA patients directly correlates with Treg cells percentages (CD4+/CD25+/CD127- cells (\%): $R=0.518$ $\mathrm{p}=0.03 ; \quad \mathrm{CD} 4+/ \mathrm{CD} 25+/$ Foxp3+ cells $(\%)$ : $\mathrm{R}=0.549, \quad \mathrm{p}=0.02$, respectively). Finally, baseline STAT3/STAT5 expression ratio on CD4+ cells > 0.93 (obtained by ROC analysis: AUC:0.754+/-0.100; Sensitivity $75.0 \%$, Specificity: $80.0 \%$ ) arose as baseline predictor factor of LDA achievement in RA patients treated with CTLA4-Ig [OR(95\%Cls): 12.0 (1.98-72.89)].

Conclusion: STAT3/STAT5 expression ratio in $\mathrm{T}$ cells at baseline identify RA patients better responding to CTLA4-Ig, which decreases Treg cells. Disclosure of Interests: Stefano Alivernini Speakers bureau: BMS, Barbara Tolusso: None declared, Anna Laura Fedele: None declared, Clara Di Mario: None declared, Luca Petricca: None declared, Maria Rita Gigante: None declared, Gianfranco Ferraccioli Speakers bureau: BMS, Roche, Elisa Gremese Consultant for: AbbVie, BMS, Celgene, Janssen, Lilly, MSD, Novartis, Sanofi, UCB, Roche, and Pfizer, Speakers bureau: BMS, Speakers bureau: Roche, Speakers bureau: AbbVie, BMS, Celgene, Jans sen, Lilly, MSD, Novartis, Sanofi, UCB, Roche, and Pfizer DOI: 10.1136/annrheumdis-2019-eular.5749

\section{SAT0074 DAS-28 DISEASE ACTIVITY DEFINES THE TNFR1/2 CO- EXPRESSION PROFILE IN RHEUMATOID ARTHRITIS PATIENTS}

Alina Alshevskaya ${ }^{1,2}$, Julia Lopatnikova ${ }^{1}$, Julia Zhukovaa ${ }^{1}$, Oksana Chumasovaa ${ }^{1}$, Nadezhda Shkaruba ${ }^{1}$, Alexey Sizikov ${ }^{1}$, Sergey Sennikov ${ }^{1,3} .{ }^{1}$ Research Institute of Fundamental and Clinical Immunology, Novosibirsk, Russian Federation; ${ }^{2}$ Biostatistics and Clinical Trials Center, Novosibirsk, Russian Federation;

${ }^{3}$ Novosibirsk State University, Novosibirsk, Russian Federation

Background: TNF-alpha act as main proinflamantory cytokine in rheumatoid arthritis (RA) immune processes. However, TNF-alpha activity and functions may be regulate not only by soluble receptors (which act as decoys) but also by number, density, and co-expression of its membranebound receptors type 1 and 2 (TNFR1 and TNFR2).

Objectives: To analyze the TNFR112 co-expression profile in RA patient with different disease activity in comparison to healthy donors (HD).

Methods: PBMC were analyzed from $46 \mathrm{HD}$ and 64 patients with RA using flow cytometry. Patients were divided according to the DAS-28 index into groups with high $(n=22,34.4 \%)$, moderate $(n=30,46.9 \%)$ and low $(n=12,18.8 \%$ ) disease activity. Co-expression of TNFR1/2 was evaluated as percentage of cell with different receptors. Number of receptors of each type per cell was counted using QuantiBrite PE beads (BD, USA). The following populations were analyzed: total monocyte pool; common pool of B lymphocytes; common pool of T lymphocytes; cytotoxic $T$ cells (CD8+), $T$ helper cells (CD4+), activated (CD25+) cells among $\mathrm{CD} 8+$ and $\mathrm{CD} 4+, \mathrm{T}$ memory (CD45R0+) and naïve $T$ cells 